\title{
FERNANDO H. LLANO ALONSO: IMPACTO DE LA MEDICINA GENÉTICA EN LOS DERECHOS DE LAS PERSONAS CON RIESGO DE TRANSMITIR ENFERMEDADES INCURABLES: UNA REVISIÓN CONCEPTUAL $Y$ NORMATIVA ${ }^{1}$
}

\section{THE IMPACT OF GENETIC MEDICINE ON THE RIGHTS OF PEOPLE AT RISK TO TRANSMIT INCURABLE DISEASES: A CONCEPTUAL AND REGULATORY REVIEW}

Resumen: En el presente trabajo pretendo realizar una aproximación a uno de los temas de investigación más novedosos en el ámbito interdisciplinar de la biomedicina, la bioética, la epigenética y el Derecho: el impacto de la medicina genética en los derechos humanos de un grupo vulnerable, el que forman los pacientes cuya herencia genética les hace potencialmente transmisores de enfermedades graves o incurables.

\begin{abstract}
In this work, I intend to analyze one of the most innovative research topics in the interdisciplinary field of biomedicine, bioethics, epigenetics and law: the impact of genetic medicine on the human rights of a vulnerable group, the patients whose genetic inheritance makes them potentially transmitters of serious or incurable diseases.
\end{abstract}

Palabras clave: Biomedicina, Bioética, Epigenética, Diagnóstico Genético Preimplantacional, Derechos Humanos

Key words: Biomedicine, Bioethics, Epigenetics, Preimplantation Genetic Diagnosis, Human Rights

\section{Planteamiento}

La perspectiva desde la que se estudia la epigenética ${ }^{2}$ (como ciencia que complementaria a la medicina genética) y su incidencia en este grupo

\footnotetext{
${ }^{1}$ Profesor Titular de Filosofía del Derecho en la Universidad de Sevilla. Artículo realizado en el marco del proyecto de excelencia financiado por el MINECO en su convocatoria RETOS de 2015 (DER201564151-R)

${ }^{2}$ Término científico (que proviene del griego epi, en o sobre, y -genética). Fue acuñado por el genetista escocés Conrad Hal Waddington, en 1942, para designar el estudio de las interacciones entre el genotipo y el fenotipo, es decir, entre la información codificada en los genes y aquella que efectivamente se expresa. El objeto de su análisis son las modificaciones en la expresión de los genes, y una de las fuentes de cambio es el factor ambiental (epimutaciones). En un artículo científico, publicado a comienzos de los años '50, Waddington explicaba con claridad el significado y alcance de la epigenética: "Parece que los cambios en el genotipo sólo tienen efectos en la evolución si traen consigo alteraciones en el proceso epigenético por el que se forman los fenotipos; y los tipos de cambio posible en el adulto o en cualquier animal están limitados a las posibles alteraciones en el sistema epigenético por el que éste se produce". Cfr., C. H. WADDINGTON, "Epigenetics and evolution", en Symposia of
} 
FERNANDO H. LLANO ALONSO: IMPACTO DE LA MEDICINA GENÉTICA EN LOS DERECHOS DE LAS PERSONAS CON RIESGO DE TRANSMITIR ENFERMEDADES INCURABLES: UNA REVISIÓN CONCEPTUAL Y NORMATIVA

FECHA DE ENVÍO DE ORIGINAL: 23.11.2016 FECHA DE ACEPTACIÓN: 25.11.2016

DOI: http://dx.doi.org/10.12795/IETSCIENTIA.2016.i02.07

vulnerable nos acerca, al mismo tiempo, a las consideraciones filosóficojurídicas realizadas por Martha C. Nussbaum en torno al derecho al desarrollo a través de su enfoque de las capacidades.

Para cumplir con este propósito dividiré mi trabajo en tres partes:

1.- En primer lugar, desde un punto de vista ético, me referiré a algunas situaciones paradigmáticas que reflejan la problemática del impacto que la medicina genética causa o puede llegar a provocar en el grupo vulnerable de la población anteriormente mencionado.

2.- En segundo lugar, desde una perspectiva iusfilosófica (o, si se prefiere jurídico, en el sentido omnicomprensivo del Derecho) haré un balance de las ventajas y los inconvenientes que supone para la dignidad y los derechos humanos el avance de la ciencia biomédica en el marco del Proyecto Epigenoma Humano. Esta cuestión nos sitúa ante el dilema de si se deben establecer límites jurídicos rígidos o flexibles a la investigación genética, en aras de la defensa de los derechos y las libertades de las personas, especialmente de aquellas que, precisamente por formar parte de grupos vulnerables, se encuentran más expuestas a sus efectos.

3.- En tercer lugar, desde un enfoque socio-político, haré una serie de consideraciones a propósito de la función de garantes que ejercen los gobiernos de los Estados miembros de la UE y las instituciones europeas en la promoción del derecho a la salud, aunque procurando siempre mantener el equilibrio con la garantía de la dignidad, las libertades y los derechos fundamentales de todos los ciudadanos dentro del Estado de Derecho (con especial atención al grupo vulnerable mencionado).

\section{La investigación genética y su repercusión en las personas con riesgo de transmitir enfermedades genéticas. Algunas consideraciones en clave ética.}

William Shakespeare afirmaba que el destino es quien baraja las cartas, pero que somos nosotros los que las jugamos. En efecto, siempre hay un componente de azar en las condiciones que nos ha tocado vivir a los seres humanos, cada individuo tiene -parafraseando al filósofo español José Ortega y Gasset- su particular circunstancia que superar. Pero conviene tener en cuenta que -como afirmaba Wilhelm Dilthey- la vida es una compleja trama de azar, destino y carácter y que, de conformidad con este juicio, por encima de las circunstancias que aleatoriamente le hayan tocado en la ruleta de la vida, el hombre está capacitado para cambiar o revertir el rumbo de los acontecimientos a su favor, y para ello no dudará en emplear los recursos técnicos y científicos que encuentre a su disposición.

Sobre la cuestión del azar, la aleatoriedad y el libre arbitrio de los seres humanos se han vertido ríos de tinta desde la Antigüedad a nuestros días, si bien el tema de la manipulación genética y su aprovechamiento para introducir

the Society for Experimental Biology VII: Evolution, Cambridge University Press, Cambridge, 1953, p. 190. 
FERNANDO H. LLANO ALONSO: IMPACTO DE LA MEDICINA GENÉTICA EN LOS DERECHOS DE LAS PERSONAS CON RIESGO DE TRANSMITIR ENFERMEDADES INCURABLES: UNA REVISIÓN CONCEPTUAL Y NORMATIVA

FECHA DE ENVÍO DE ORIGINAL: 23.11.2016 FECHA DE ACEPTACIÓN: 25.11.2016.

DOI: http://dx.doi.org/10.12795/IETSCIENTIA.2016.i02.07

mejoras en la naturaleza humana ha sido recurrente a lo largo del siglo XX, tanto en el ámbito de la literatura (Aldous Huxley, George Orwell), como en el de la ciencia (desde que en 1944 Oswald Theodore Avery, Colin MacLeod y Maclyn McCarty aislaran ADN como material genético, hasta la publicación en 2003 por parte del Proyecto Genoma Humana de la prmera secuenciación completa del mapa genético humano), y también en el mundo de la filosofía (Francis Fukuyama y Jürgen Habermas).

Precisamente el último de estos autores, el pensador alemán Jürgen Habermas (discípulo aventajado de Max Horkheimer y Theodor Adorno, dos de los máximos representantes de la Escuela de Frankfurt) publicaba, a comienzos del siglo XXI, un libro titulado: El futuro de la naturaleza humana (2001) en el que planteaba el siguiente dilema a propósito de los retos que plantea la ingeniería genética de nuestro tiempo:

"¿Queremos contemplar la posibilidad categorialmente nueva de intervenir en el genoma humano como un incremento de libertad necesitado de regulación normativa o como una autoinvestidura de poderes para llevar a cabo unas transformaciones que dependan de las preferencias y no necesiten ninguna autolimitación? Solo cuando esta pregunta fundamental se haya resuelto a favor de la primera alternativa podrán debatirse las fronteras de una eugenesia negativa, cuya meta sea, sin malentendidos, eliminar males"3.

La cuestión que formula Habermas apunta a la descodificación del genoma humano y al cambio de paradigma que se ha producido en el pensamiento secular de la modernidad europea, que tradicionalmente ha coincidido con la fe religiosa en la concepción de la fecundación como un proceso natural contingente e indisponible cuya consecuencia es una combinación imprevisible de dos secuencias cromosómicas distintas. Según el paradigma ético generalmente compartido en Europa hasta fechas recientes, las condiciones orgánicas de partida del embrión humano se sustraerían a la manipulación genética y a la programación intencionada de otras personas (incluso de sus progenitores). Ciertamente, se ha operado un cambio en ese paradigma como consecuencia de la descodificación del mapa genético humano a principios del siglo XX, abriéndose nuevas vías de investigación en el desarrollo de la investigación científica contra enfermedades consideradas hasta ahora incurables, sino también en la selección de la dotación genética de nuestros descendientes, lo cual ha planteado no pocas controversias de carácter ético-científico entre partidarios y detractores de las técnicas de manipulación genética que, en última instancia, ponen en tela de juicio la autorrealización espontánea y la libertad ética de las personas sobre las que se actúa sin contar con su previo consentimiento.

El dilema se les plantea frecuentemente a aquellos padres que desean evitar la transmisión de enfermedades hereditarias, y a quienes se ofrece el

\footnotetext{
${ }^{3}$ J. HABERMAS, El futuro de la naturaleza humana ¿Hacia una eugenesia liberal?, trad. esp:

C. S. Paidós, Barcelona-Buenos Aires-México, 2002, pp. 24-25.
} 
FERNANDO H. LLANO ALONSO: IMPACTO DE LA MEDICINA GENÉTICA EN LOS DERECHOS DE LAS PERSONAS CON RIESGO DE TRANSMITIR ENFERMEDADES INCURABLES: UNA REVISIÓN CONCEPTUAL Y NORMATIVA

FECHA DE ENVÍO DE ORIGINAL: 23.11.2016 FECHA DE ACEPTACIÓN: 25.11.2016.

DOI: http://dx.doi.org/10.12795/IETSCIENTIA.2016.i02.07

diagnóstico genético preimplantacional (DGP), que consiste en el diagnóstico de alteraciones genéticas y cromáticas en los embriones, antes de su implantación, para lograr que los niños nazcan libres de enfermedades hereditarias. La técnica del DGP permite aislar los embriones en estadio octocelular en una prueba preventiva en la que, llegado el caso, el embrión examinado en el tubo de ensayo no se volvería a implantar, de manera que se evitaría que la madre tuviera que interrumpir su embarazo que, de otro modo, habría que aplicar tras el diagnóstico prenatal ${ }^{4}$.

Otro motivo de controversia surgido entre científicos, teólogos y filósofos es el suscitado en torno a las técnicas transhumanistas, que permiten la generación de embriones con el ADN de tres personas diferentes a fin de evitar enfermedades mitocondriales ${ }^{5}$. La finalidad de esta técnica transhumanista que no deja de ser una manipulación de los embriones- es la de evitar que las mutaciones genéticas se transmitan a los niños utilizando el ADN de una persona para que el niño nazca sano ${ }^{6}$. A propósito de las nuevas tecnologías aplicadas a la ciencia biomédica, con idea de aumentar las capacidades físicas del ser humano y mejorar sus características genéticas a través de la nanotecnología, la regeneración celular, el implante de dispositivos que interactúan con el cerebro..., algunos teólogos cristianos se han preguntado si ese supuesto perfeccionamiento de la especie humana, si esa evolución genética que parece realizar el mito del super-hombre y es obtenida a partir de los ensayos y experimentos realizados en el ámbito de la epigenética, es

\footnotetext{
${ }^{4}$ S. FRANKLIN y C. ROBERTS, Born and Made. An Ethnography of Preimplantation Genetic Diagnosis, Princeton University Press, Princeton/Oxford, 2006, pp. 25 y ss.
}

${ }^{5}$ En Febrero de 2015 el Parlamento británico aprobó una ley que autoriza la reproducción asistida que utiliza el ADN de tres personas para prevenir enfermedades hereditarias graves. Según los defensores de esta revolucionaria técnica reproductiva, las mutaciones de ADN en las mitocondrias son bastante comunes: según datos oficiales del NHS de 2013, uno de cada 200 bebés era portador en el Reino Unido de una mutación mitocondrial, transmitiéndose de madre a hijo, que potencialmente puede causar desde problemas neurológicos, musculares 0 cardiacos, hasta sordera o diabetes; además, uno de cada 6500 bebés está seriamente afectado por los efectos de esta mutación y, como consecuencia de ella, podría sufrir enfermedades mortales para las que no hay aún curación. Los detractores de esta técnica, la Iglesia de Inglaterra y la Iglesia Católica, aducen que implica la destrucción del embrión humano, y con su puesta en práctica se podría abrir la puerta a futuras modificaciones genéticas en los embriones.

${ }^{6}$ Frente a la eugenesia, práctica científica que consiste en una búsqueda de los mejores genes (y que durante el nazismo provocó el exterminio de millares de personas para eliminar "rasgos indeseables"), el transhumanismo -técnica adoptada recientemente en Inglaterra- consiste en la aplicación de procedimientos científicos para aumentar las capacidades físicas, intelectuales y emocionales de los seres humanos. Cfr., R. Dimond, "Social and ethical issues in mitochondrial donation", British Medical Bulletin, 115 (1), 2015, pp. 173-182.

También disponible en versión digital: http://bmb.oxfordjournals.org/content/115/1/173.full 
FERNANDO H. LLANO ALONSO: IMPACTO DE LA MEDICINA GENÉTICA EN LOS DERECHOS DE LAS PERSONAS CON RIESGO DE TRANSMITIR ENFERMEDADES INCURABLES: UNA REVISIÓN CONCEPTUAL Y NORMATIVA

FECHA DE ENVÍO DE ORIGINAL: 23.11.2016 FECHA DE ACEPTACIÓN: 25.11.2016.

DOI: http://dx.doi.org/10.12795/IETSCIENTIA.2016.i02.07

suficiente para formar a la persona como sujeto moral y, lo que es aún más importante, si sirve para completar su desarrollo espiritual ${ }^{7}$.

La evolución humana ha pasado por al menos por tres fases. Hasta hace pocas décadas la evolución se entendía como adaptación natural al medio por parte del ser humano; más tarde, en una fase intermedia, se pasa a una fase de adaptación tecnológica a través de medios artificiales (prótesis, implantes y otros medios auxiliares que nos procuran calidad de vida, salud y bienestar); en la actualidad, hemos pasado a una tercera fase de evolución autodirigida a perfilar los resultados de nuestra interacción con el medioambiente en el que vivimos, con lo cual no solo hemos llegado a configurarnos nosotros mismos, sino que también podemos permitirnos incluso a programar la mejora de nuestros propios organismos ${ }^{8}$.

Aunque la premisa de partida de esta evolución autodirigida es la búsqueda de la felicidad (en la medida en que mejora cualitativamente nuestra composición genética y, con ello, el aumento de las expectativas de vida), cabría preguntarse, desde un punto de vista ecológico, en qué posición queda la relación del hombre con la naturaleza. Un aumento de las expectativas de vida del hombre nos sitúa ante la cuestión de la superpoblación del planeta y la explotación de los recursos naturales (que no son inagotables). Contemplada esta cuestión sobre el desarrollo tecnológico y su efecto en la sociedad moderna desde un punto de vista ético-filosófico, surge una duda -que en el siglo pasado ya fue motivo de reflexión por pensadores como Spengler ${ }^{9}$, Heidegger ${ }^{10}$ y Ortega $^{11}$ - en torno a la relación del hombre con la técnica: ¿se ha invertido la relación de dominio entre el hombre y la técnica? ¿ha pasado el hombre (el hombre biónico) de una posición inicial de dominio a una situación de dependencia (o incluso subordinación) respecto a la ciencia y la técnica?

\section{Medicina genética, dignidad humana y derechos humanos. Claves para un debate iusfilosófico}

\footnotetext{
${ }^{7}$ R. COLE-TURNER, "The Transhumanist Challenge", en Transhumanism and Trascendence: Christian Hope in an Age of Technological Enhancement (ed: R. COLE-TURNER), Georgetown University Press, Washington D.C., 2011, pp. 1-18.
}

${ }^{8}$ M. C. CROW, "Science, Technology and Democracy, en Beyond Humanism: Trans- and Posthumanism (ed: S. L. SORGNER). Vol III: Building Better Humans? Refocusing the Debate on Transhumanism (ed: H. TIROSH-SAMUELSON and K. L. MOSSMAN), Peter Lang, Frankfurt am Main/Berlin/Bern/Bruxelles/New York/Oxford/Wien, pp. 13 y ss.

${ }^{9}$ O. SPENGLER, El hombre y la técnica (1931), trad. esp: M. García Morente, Espasa-Calpe, Madrid, 1967.

${ }^{10}$ M. HEIDEGGER, "El final de la filosofía y la tarea de pensar", en Tiempo y ser (1962), trad. esp: J. L. Molinuevo, Tecnos, Madrid, 1999.

${ }^{11}$ J. ORTEGA Y GASSET, "Meditación de la técnica" (1939), Obras completas (V), Fundación José Ortega y Gasset/Taurus, Madrid, 2004-2010, pp. 551-605. Véase también: "El mito del hombre allende la técnica" (1952), Obras completas (VI), cit., pp. 811-817. Cfr., F. H. LLANO ALONSO, "El hombre y la técnica en Ortega y Gasset", lus et Scientia, 1 (2015), pp. 1-24. 
FERNANDO H. LLANO ALONSO: IMPACTO DE LA MEDICINA GENÉTICA EN LOS DERECHOS DE LAS PERSONAS CON RIESGO DE TRANSMITIR ENFERMEDADES INCURABLES: UNA REVISIÓN CONCEPTUAL Y NORMATIVA

FECHA DE ENVÍO DE ORIGINAL: 23.11.2016 FECHA DE ACEPTACIÓN: 25.11.2016.

DOI: http://dx.doi.org/10.12795/IETSCIENTIA.2016.i02.07

Como se ha puesto de manifiesto en el anterior epígrafe, el desarrollo de la ciencia biomédica en general, y de la epigenética en particular, ha supuesto un avance en la mejora de la calidad y de las expectativas de vida de los seres humanos. Pero esta evolución de las técnicas de ingeniería genética supone, en cierto modo, una instrumentalización de la persona (concebida como sujeto moral) que relativiza y subvierte el sentido sagrado del concepto de dignidad humana, inmortalizado por Kant en la máxima del imperativo categórico que establece lo siguiente:

"Obra de tal modo que uses la humanidad, tanto en tu persona como en la persona de cualquier otro, siempre como un fin al mismo tiempo y nunca solamente como un medio"12.

Con estas palabras Kant nos manifiesta su convicción de que la humanidad es res sacra, como sujeto de una ley moral que se funda a su vez en la autonomía de la voluntad (una voluntad que debe entenderse como voluntad libre y no mediatizada). Como ha señalado Antonio E. Pérez Luño en un reciente estudio sobre "Kant y los derechos humanos", la dignidad humana entraña no sólo la garantía negativa de que la persona no va a ser objeto de ofensas y humillaciones, sino que supone también la afirmación positiva del pleno desarrollo de la personalidad de cada individuo. Según este autor,

"El pleno reconocimiento de la total autodisponibilidad, sin interferencias o impedimentos externos, de las posibilidades de actuación propias de cada hombre; de otro, la autodeterminación que surge de la libre proyección histórica de la razón humana, antes que de una predeterminación dada por la naturaleza de una vez por todas"13.

Desde la óptica liberal que inspira la teoría de los derechos humanos, las nuevas tecnologías en las que se apoya la ingeniería genética, plantean un dilema ético-jurídico entre la indisponibilidad natural y espiritual del ser humano desde su origen natural, y la libertad que tiene el individuo de elegir su configuración genética, frente al intervencionismo estatal, y en aras de su salud y bienestar. Por otra parte, esta confianza inquebrantable en la ciencia y el desarrollo técnico que -según Habermas- "va más allá de la aplicación de las terapias genéticas, y llega hasta el shopping in the genetic supermarket"14, también puede suponer un conflicto intergeneracional entre los padres y sus hijos y descendientes.

\footnotetext{
${ }^{12}$ I. KANT, Fundamentación de la metafísica de las costumbres (1785), trad. esp: M. García Morente, Espasa-Calpe, Madrid, 1921, p. 70. Véase también: Crítica de la razón práctica (1788), trad. esp: E. Miñana y Villagrasa y M. García Morente, Sígueme, Salamanca, 1998, pp. 111 y 162.

13 A. E. PÉREZ LUÑO, "Kant y los derechos humanos", Historia de los derechos fundamentales. Tomo II: Siglo XVIII. Volumen II: La filosofía de los derechos humanos (Dir: G. PECES-BARBA MARTÍNEZ, E. FERNÁNDEZ GARCÍA y R. DE ASÍS ROIG), Dykinson-Instituto de Derechos Humanos "Bartolomé de Las Casas. Universidad Carlos III de Madrid, Madrid, 2001 , p. 471.

${ }^{14}$ J. HABERMAS, El futuro de la naturaleza humana ¿ Hacia una eugenesia liberal?, cit., p. 102.
} 
FERNANDO H. LLANO ALONSO: IMPACTO DE LA MEDICINA GENÉTICA EN LOS DERECHOS DE LAS PERSONAS CON RIESGO DE TRANSMITIR ENFERMEDADES INCURABLES: UNA REVISIÓN CONCEPTUAL Y NORMATIVA

FECHA DE ENVÍO DE ORIGINAL: 23.11.2016 FECHA DE ACEPTACIÓN: 25.11.2016.

DOI: http://dx.doi.org/10.12795/IETSCIENTIA.2016.i02.07

En este sentido, cabe distinguir dos líneas de pensamiento: en primer lugar, desde una perspectiva utilitarista o consecuencialista se deja a los padres que decidan sobre la composición de la disposición genética de sus hijos, sin que se tengan que someter a ninguna regulación estatal. A este respecto, la técnica genética amplia el derecho de reproducción y las competencias materiales de la patria potestad, de manera que el particular puede hacer valer estos derechos individuales fundamentales ante el Estado. Frente a este enfoque utilitarista o consecuencialista, se sitúa la posición deontológica y kantiana que apuesta por el ser humano como un fin en sí mismo, por lo que será el Estado de Derecho quien garantiza la protección de los derechos y libertades del individuo (incluyendo al no nacido). El control del Estado en la protección de la inviolabilidad e indisponibilidad del no nacido (incluso en su estado embrionario) no debe entenderse como una contradicción en la perspectiva liberal katiana, sino como una garantía de los derechos y libertades fundamentales de las futuras generaciones a través del ordenamento jurídico y la tutela de los poderes públicos ${ }^{15}$.

En relación con el embrión humano y el feto, se ha debatido sobre la procedencia de la distinción teórico-conceptual -como propone Habermasentre la "inviolabilidad" de la vida humana (que para él tiene un valor absoluto y es propio de las personas ya nacidas), y la "indisponibilidad" de la vida del feto y el embrión, pues -para el filósofo germano- este último es un concepto más relativo, en la medida en que -en su opinión- dicha inviolabilidad debe plantearse desde una concepción moral gradual. Frente a esta tesis intermedia de Habermas, hay otras dos posturas más extremas y antagónicas: de un lado está la posición de la Iglesia Católica y Protestante que aboga por la realidad personal del embrión "in vitro", es decir, prácticamente desde el momento en que el óvulo ha sido fecundado; y, en el lado contrario, se encuentra la concepción naturalista -más alineada con la doctrina utilitarista anglosajonaque considera al embrión como un conjunto de células que no tienen un valor superior ni diferente al que tienen otras células humanas (uno de los defensores más radicales de esta tesis es el filósofo australiano Peter Singer, para quien la vida del embrión humano no tiene más valor que la que pueda tener cualquier otro embrión animal) ${ }^{16}$.

\subsection{El Diagnóstico Genético Preimplantacional: el dilema entre la libertad reproductiva de los padres y la protección de las barreras de la identidad genética de las futuras generaciones}

Al hilo de la controversia sobre el status del embrión, uno de los ejemplos más ilustrativos del difícil tratamiento jurídico que ha recibido hasta la fecha la medicina genética ("también conocida como "medicina personalizada" o " a la carta") es el DGP. En términos genéricos, el DGP supone un avance en la

\footnotetext{
${ }^{15}$ En línea con este argumento, Ernst BLOCH afirma: "Nadie vive porque quiere. Pero, después de que se vive, hay que querer seguir viviendo. $Y$ hay que seguir fiel a ello, si se quiere llegar a algo". Cfr., Derecho y dignidad humana, trad.: F. González Vicén, Aguilar, Madrid, 1980, p. 4.

${ }^{16}$ P. SINGER, Una vida ética. Escritos, trad. esp: P. de Lora del Toro, Taurus, Madrid, 2002, pp. 193-198.
} 
FERNANDO H. LLANO ALONSO: IMPACTO DE LA MEDICINA GENÉTICA EN LOS DERECHOS DE LAS PERSONAS CON RIESGO DE TRANSMITIR ENFERMEDADES INCURABLES: UNA REVISIÓN CONCEPTUAL Y NORMATIVA

FECHA DE ENVÍO DE ORIGINAL: 23.11.2016 FECHA DE ACEPTACIÓN: 25.11.2016

DOI: http://dx.doi.org/10.12795/IETSCIENTIA.2016.i02.07

detección, el diagnóstico y el tratamiento de enfermedades derivado del conocimiento y la comprensión de la estructura genética de un individuo concreto. En términos específicos, el DGP consiste en una técnica diagnóstica que sirve para comprobar la "salud genética" de un embrión vivo, "in vitro", antes de que se decida si va a ser implantado en el útero materno o si, por el contrario, no cumple con su finalidad procreativa $y$, por ende, debe ser descartado para evitar el nacimiento de niños con enfermedades hereditarias graves.

Pero el DGP presenta algunos claroscuros que conviene comentar, por su especial repercusión en el avance de la biomedicina, pero también por los probables riesgos que ésta entraña, en la medida en que se traspasen límites éticos y jurídicos hasta ahora infranqueables. A este respecto, como ha advertido Fernando Abellán, el DGP no se presenta solamente como un instrumento útil para luchar contra la transmisión de patologías hereditarias, sino que también despliega un amplio abanico de opciones y posibilidades para los seres humanos que algunos valoran como una gran oportunidad para el desarrollo de la libertad reproductiva de los padres que desean garantizarle a sus hijos las mejores condiciones de salud, aunque, al mismo tiempo, ha suscitado en otros la desconfianza y el temor propios de quienes creen ver en el desbordamiento ético-jurídico de la medicina genomizada, y particularmente de técnicas epigenéticas como el DGP, el retorno del fantasma de la eugenesia. En resumidas cuentas, para algunos, el DGP ha contribuido al surgimiento de un nuevo concepto de paternidad responsable que se vale de la información genética para decidir sobre sus opciones reproductivas y, para otros, en una forma de "neoeugenesia" que tiene su origen en la libertad reproductiva de los individuos o de las parejas, a diferencia de la eugenesia clásica (producto de políticas estatales de signo totalitario) ${ }^{17}$.

En última instancia, el problema a resolver es de carácter ético-jurídico. El legislador y los jueces en la práctica deben decidir dónde situar el punto de equilibrio entre la libre voluntad de los progenitores y la esfera de protección de la identidad genética de las futuras generaciones. Asimismo, como veremos en el último epígrafe, también habría que determinar qué papel le corresponde a la sociedad, a través del Estado, en el acotamiento de la libertad reproductiva. A continuación voy a hacer una breve referencia a cómo se regula en España esta cuestión en la Ley 14/2006, de 26 de mayo, sobre las técnicas de reproducción humana asistida, y también aludiré a la situación normativa del DGP en el Derecho comparado.

\subsection{La situación legal del DGP en España y otros países europeos}

\subsubsection{La Ley española sobre técnicas de reproducción humana asistida}

\footnotetext{
${ }^{17}$ F. ABELLÁN, "Aspectos bioéticos y legales del diagnóstico genético preimplantatorio (DGP)", Revista Iberoamericana de Fertilidad, 23/2006, p. 124.
} 
FERNANDO H. LLANO ALONSO: IMPACTO DE LA MEDICINA GENÉTICA EN LOS DERECHOS DE LAS PERSONAS CON RIESGO DE TRANSMITIR ENFERMEDADES INCURABLES: UNA REVISIÓN CONCEPTUAL Y NORMATIVA

FECHA DE ENVÍO DE ORIGINAL: 23.11.2016 FECHA DE ACEPTACIÓN: 25.11.2016.

DOI: http://dx.doi.org/10.12795/IETSCIENTIA.2016.i02.07

La Ley 14/2006 tiene por objeto la regulación de los supuestos y requisitos de utilización de gametos y preembriones humanos crioconservados (art. 11), si bien, antes de proceder a desarrollar esa casuística, aclara en el art.1.2 el significado del concepto de preembrión in vitro: "constituido por el grupo de células resultantes de la división progresiva del ovocito desde que es fecundado hasta 14 días más tarde".

En el art. 3.1 el legislador establece el derecho de la mujer receptora de las técnicas de reproducción asistida a estar informada de sus posibilidades de éxito, así como de sus riesgos y de las condiciones de dicha aplicación. La aceptación de la aplicación de técnicas de reproducción asistida y diagnóstico preimplantacional requieren el consentimiento informado de la mujer receptora, quien podrá pedir en cualquier momento anterior a la transferencia embrionaria que se suspenda el proceso (art. 3.4 y 3.5 ).

En relación con las técnicas de diagnóstico preimplantacional la Ley 14/2006 permite su práctica en centros de reproducción asistida autorizados solamente cuando se realiza para la detección de enfermedades hereditarias graves, de aparición precoz y no susceptibles de tratamiento curativo posnatal $\mathrm{u}$ otras alteraciones que puedan comprometer la viabilidad del preembrión (cromosopatías fundamentalmente), a fin de llevar a cabo la selección de los preembriones no afectos para su implantación (art. 12.1). En estos casos, la aplicación de las técnicas de diagnóstico preimplantacional se comunicará a la autoridad sanitaria competente quien, a su vez, informará de ella a la Comisión Nacional de Reproducción Humana Asistida (CNRHA).

La nueva ley no excluye la aplicación de técnicas de diagnóstico preimplantacional a otras situaciones e incluso permite el DGP extensivo, o con finalidad terapéutica para terceros, pero los condiciona a la autorización expresa, caso a caso, de la autoridad sanitaria correspondiente, previo informe favorable de la CNRHA, que deberá evaluar la características clínicas, terapéuticas y sociales de cada caso (art. 12.2).

La regulación del DGP no está armonizada a nivel europeo al no existir una norma supranacional sobre esta materia. Así pues, para conocer la realidad jurídica de las técnicas de diagnóstico preimplantacional en los países de nuestro entorno, es preciso estudiar la legislación nacional especializada de cada país miembro de la UE y, desde una perspectiva comparatista, llegar conclusiones parciales que, una vez sumadas, nos permitan tener una visión global de este mosaico jurídico ${ }^{18}$. A continuación voy a hacer una referencia selectiva al tratamiento que algunos de estos países han hecho del DGP y que, bien sea por su flexibilidad o por su carácter restrictivo, me han llamado más la atención.

\subsubsection{La Ley portuguesa}

\footnotetext{
${ }^{18}$ I. ALKORTA IDIAKEZ, Regulación jurídica de la medicina reproductiva. Derecho español y comparado, Aranzadi, Cizur Menor (Navarra), 2003, pp. 316-321.
} 
FERNANDO H. LLANO ALONSO: IMPACTO DE LA MEDICINA GENÉTICA EN LOS DERECHOS DE LAS PERSONAS CON RIESGO DE TRANSMITIR ENFERMEDADES INCURABLES: UNA REVISIÓN CONCEPTUAL Y NORMATIVA

FECHA DE ENVÍO DE ORIGINAL: 23.11.2016 FECHA DE ACEPTACIÓN: 25.11.2016.

DOI: http://dx.doi.org/10.12795/IETSCIENTIA.2016.i02.07

La Ley 32/2006, de 26 de julio regula el DGP en su Capítulo V (arts. 28 y 29). Permite la aplicación de técnicas de diagnóstico preimplantacional dirigidas a detectar los embriones que no sean portadores de anomalías graves antes de ser transferidos al útero de la mujer. La ley portuguesa prohíbe expresamente la utilización del DGP para la detección (y selección) del sexo del futuro bebé, excepto cuando exista un riesgo elevado de enfermedad genética grave ligada al sexo (arts. 7.2 y 7.3). Por último, la aplicación de las técnicas de diagnóstico preimplantacional debe realizarse bajo la supervisión del médico especialista responsable, y su autorización depende del reconocimiento por parte del Conselho Nacional de Procriação medicamente Assistida de su valor científico para el diagnóstico, el tratamiento o la prevención de enfermedades genéticas graves (art. 28.3). La Ley 32/2006 tiene como principal peculiaridad el hecho de priorizar expresamente la práctica del DGP a personas que, por herencia genética, tienen un alto riesgo de contraer y transmitir a sus descendientes enfermedades que producen la muerte precoz 0 dolencias graves.

\subsubsection{La Ley italiana}

La Ley 40/2004, de 19 de febrero no se refiere expresamente al DGP, pero prohíbe en términos generales la experimentación sobre cualquier embrión humano (art. 13.3 a). Tan solo autoriza la investigación clínica y experimental con la condición de que se lleven a cabo con una finalidad terapéutica y diagnóstica en aras de la salud y el desarrollo del embrión humano (13.2). Lo más llamativo de esta ley es que, en su art. 13.3 b, alude al DGP cuando prohíbe la aplicación de cualquier técnica de medicina genética. En dicho parágrafo el legislador presume la finalidad eugenésica que persigue la selección, manipulación o cualquier otro procedimiento artificial dirigidos tanto a alterar el patrimonio genético del embrión o del gameto, como a predeterminar sus características genéticas de manera artificial.

\subsubsection{La Ley francesa}

El Code de la santé publique, en los arts. R2131-22-1 a 2131-26-3 de la Sección 3 del Título III del Libro I de la Segunda parte, define y regula las condiciones de realización del $\mathrm{DGP}^{19}$. Permite muy restrictivamente la aplicación de las técnicas de diagnóstico preimplantacional, en casos de alto riesgo de transmisión de enfermedades graves, y establece estrictos controles para la práctica del DGP. La novedad de la normativa francesa estriba en el hecho de que autoriza el DGP extensivo, de forma experimental, y bajo condiciones muy rigurosas: que la pareja haya tenido un niño que padezca una enfermedad genética que conlleve la muerte durante los primeros años de vida y que esté reconocida como incurable en el momento del diagnóstico; que el pronóstico vital de ese niño pueda ser mejorado, de forma decisiva, aplicándole una terapéutica que no comporte un menoscabo de la integridad del cuerpo del

\footnotetext{
${ }^{19}$ El Código de Salud Pública ha sido parcialmente reformado por la Ley relativa a la bioética 2004/800, de 6 de agosto (arts. 25-27) y, posteriormente, por la La Ley 2011-814 relativa a la bioética, de 7 de julio (arts. 20 y 22).
} 
FERNANDO H. LLANO ALONSO: IMPACTO DE LA MEDICINA GENÉTICA EN LOS DERECHOS DE LAS PERSONAS CON RIESGO DE TRANSMITIR ENFERMEDADES INCURABLES: UNA REVISIÓN CONCEPTUAL Y NORMATIVA

FECHA DE ENVÍO DE ORIGINAL: 23.11.2016 FECHA DE ACEPTACIÓN: 25.11.2016.

DOI: http://dx.doi.org/10.12795/IETSCIENTIA.2016.i02.07

ser que vaya a nacer (a partir del embrión que se transfiera al útero materno), etc.

\subsubsection{El sistema británico}

La investigación sobre la fertilización y la experimentación con embriones humanos se regula en el Reino Unido por la Human Fertilization and Embryology Act de 1990. Esta ley ha creado una autoridad administrativa, la Human Fertilization and Embryology Authority (en adelante HFEA), que tiene competencia para autorizar y supervisar las peticiones de diagnóstico de cada nueva patología, y para conceder licencias a los centros que desean llevar a la práctica técnicas de diagnosis genética preimplantacional. Recientemente, la HFEA ha publicado un catálogo on-line con los genes humanos y las condiciones genéticas susceptibles de ser diagnosticadas mediante el DGP en clínicas autorizadas ${ }^{20}$. Por último, para que la HFEA autorice el DGP debe estudiar cada caso y determinar si existen condiciones genéticas serias que justifiquen este test. Además de obtener la autorización administrativa de la HFEA, las clínicas están obligadas a comprobar que el DGP es el tratamiento más adecuado para el paciente.

\subsubsection{La Ley alemana}

EI DGP está prohibido por la Ley sobre protección de embriones de 1990, la Embryonenschutzgesetz-ESchG, de 13 de diciembre ${ }^{21}$. La ley sanciona con tres años de prisión a quien utilice un embrión humano por cualquier razón que no sea la de su conservación. Lo característico de esta norma es que considera como embrión tanto al óvulo humano fecundado (susceptible de desarrollo a partir de la fusión de los núcleos) como a cualquier célula totipotente extraída de un embrión susceptible de desarrollarse hasta convertirse en un individuo. Como ha observado Fernando Abellán, hasta la fecha se han rechazado todas las tentativas de legalizar el DGP debido a que, según los críticos de esta técnica avanzada de la medicina genética, contraviene el derecho a la protección del embrión y porque esta práctica supone un riesgo de favorecer tendencias eugenésicas ${ }^{22}$.

\section{La Unión Europea, los Estados y la sociedad contemporánea ante los retos de la medicina genética}

\subsection{La política sanitaria de la Unión Europea}

La heterogeneidad legislativa en materia de medicina genética existente en los países miembros de la Unión Europea (en adelante UE) supone obstáculo en el avance hacia la integración de un sistema sanitario europeo y, en todo caso, retrasa el cumplimiento los objetivos de la estrategia general sanitaria

\footnotetext{
${ }^{20}$ Online Mendelian Inheritance in Man (OMIM): http://guide.hfea.gov.uk/pgd/ (web oficial de a HFEA consultada en junio de 2016).

${ }^{21}$ Arts. 1.2 y 8.1 de la Embryonenschutzgesetz-ESchG.

${ }^{22}$ F. ABELLÁN, Selección genética de embriones: entre la libertad reproductiva y la eugenesia, Comares, Granada, 2007, p. 56.
} 
FERNANDO H. LLANO ALONSO: IMPACTO DE LA MEDICINA GENÉTICA EN LOS DERECHOS DE LAS PERSONAS CON RIESGO DE TRANSMITIR ENFERMEDADES INCURABLES: UNA REVISIÓN CONCEPTUAL Y NORMATIVA

FECHA DE ENVÍO DE ORIGINAL: 23.11.2016 FECHA DE ACEPTACIÓN: 25.11.2016.

DOI: http://dx.doi.org/10.12795/IETSCIENTIA.2016.i02.07

Europa $2020^{23}$. Como reconoce la propia Comisión en su informe "Investing in Health", publicado en febrero de 2013, la salud es un valor en sí mismo e invertir en ella constituye una precondición necesaria para la prosperidad económica de los países miembros, la cohesión social y el incremento de la productividad en el trabajo (en la medida en que se mejoran sustancialmente las expectativas de vida sana de sus ciudadanos) ${ }^{24}$.

Aunque los gobiernos nacionales son los responsables de organizar la asistencia sanitaria y garantizar su prestación, la UE ejerce un papel complementario ayudando a los gobiernos nacionales a alcanzar los objetivos comunes, poniendo en común recursos y abordando retos compartidos entre todos sus miembros. La política sanitaria de la UE, aplicada a través de su Estrategia Sanitaria, se centra en cinco objetivos concretos:

- La prevención, sobre todo a través del fomento de estilos de vida más saludables.

- La igualdad de oportunidades para todos por lo que respecta a la buena salud y la asistencia sanitaria de calidad.

- La lucha contra las amenazas graves para la salud que afecta a algunos países de la UE.

- El envejecimiento con buena salud.

- El apoyo a los sistemas de salud dinámicos y las nuevas tecnologías ${ }^{25}$.

Para favorecer la consecución de estos objetivos, la UE prevé una actuación específica a través de la elaboración de normativa sobre pacientes, productos y servicios sanitarios; financia proyectos sanitarios a través del Programa de Salud de la UE; impulsa la cooperación entre los sistemas sanitarios de los países miembros y el establecimiento en ellos de las mejores prácticas (mediante actividades de promoción de la salud, detección de factores de riesgo, gestión de las enfermedades y sistemas sanitarios, etc.)

La vocación de la UE por la cooperación sanitaria entre los estados miembros se pone de relieve en la creación de las European reference networks (Redes Europeas de Referencia, en adelante RER), por las que se crea una estructura de gobierno para el intercambio de conocimientos e información entre los profesionales sanitarios europeos, así como la coordinación de la asistencia en todo el territorio de la UE. En el art. 12 de la Directiva 2011/24/EU, de 9 de marzo sobre los derechos de los pacientes en la asistencia sanitaria transfronteriza, se detallan los principales objetivos a los

\footnotetext{
${ }^{23}$ Cfr., http://ec.europa.eu/europe2020/index en.htm (Web oficial de la Comisión Europea consultada en junio de 2016).

${ }^{24}$ Cfr., http://ec.europa.eu/health/strategy/docs/swd investing in health.pdf (Web oficial de la Comisión Europea consultada en junio de 2016).

${ }^{25} \mathrm{Cfr}$., http://europa.eu/pol/health/index es.htm (Web oficial de la Unión Europea consultada en junio de 2016).
} 
FERNANDO H. LLANO ALONSO: IMPACTO DE LA MEDICINA GENÉTICA EN LOS DERECHOS DE LAS PERSONAS CON RIESGO DE TRANSMITIR ENFERMEDADES INCURABLES: UNA REVISIÓN CONCEPTUAL Y NORMATIVA

FECHA DE ENVÍO DE ORIGINAL: 23.11.2016 FECHA DE ACEPTACIÓN: 25.11.2016.

DOI: http://dx.doi.org/10.12795/IETSCIENTIA.2016.i02.07

que se dirigen las RER: el fomento de las innovaciones en ciencia médica y tecnologías de salud (12.2.a), la puesta en común de conocimientos en prevención de enfermedades (12.2.b), facilitar el desarrollo en el diagnóstico de pacientes (12.2.c) $)^{26}$.

4.2El papel de los Estados miembros de la UE ante el avance de las técnicas de la medicina genética

En el apartado anterior hemos comprobado cómo la UE apoya a los Estados miembros a colaborar entre sí sobre la base los valores y principios sanitarios europeos ${ }^{27}$. Pero, como hemos podido comprobar en el epígrafe 3, en materia sanitaria la realidad jurídica de cada uno de los estados miembros es distinta y permite regular con matices, cuando no con notables diferencias en temas como la sanidad electrónica (aplicación de las TIC's a la investigación médica), la medicina genética, etc. Sin embargo, es a los gobiernos de los respectivos países miembros de la UE a quienes les corresponde gestionar sus propios sistemas de salud.

La primera conclusión a la que se llega tras comparar los sistemas sanitarios en los países de la UE, tanto en sus características como en los diversos indicadores de salud, es que existe una absoluta asimetría en marcadores tan relevantes como: su sistema de financiación (unos lo hacen a través de cotizaciones obligatorias y otros a través de los impuestos); la provisión de servicios y los diferentes mecanismos de participación de los ciudadanos en los costes; las medidas tomadas para proteger a los colectivos más vulnerables (población con bajos ingresos y en desventaja socioeconómica, enfermos crónicos y graves, discapacitados, ancianos, mujeres embarazadas y población infantil) $)^{28}$.

La segunda conclusión que permite extraer este panorama tan heterogéneo de sistemas sanitarios en las distintas legislaciones y prácticas nacionales es que, aunque todos los Estados miembros de la UE cumplen con lo establecido en la Carta de los derechos fundamentales de la Unión Europea sobre la Seguridad social y ayuda social (art. 34) y la protección de la salud (art. 35), existe una gran disonancia exegética por parte de los legisladores de los países de la UE respecto a la interpretación del principio de inviolabilidad de la dignidad humana (art. 1) y al derecho a la integridad de la persona (art. 3$)^{29}$.

26

Cfr.,

http://eurlex.europa.eu/LexUriServ/LexUriServ.do?uri=OJ:L:2011:088:0045:0065:es:PDF (Web del Diario Oficial de la Unión Europea, 4-4-2011, consultada en junio de 2016)

${ }^{27}$ DO C 146 de 22.6.2006, p. 1.

${ }^{28}$ Ministerio de Sanidad, Servicios Sociales e Igualdad, "Los sistemas sanitarios en los Países de la UE: características e indicadores de salud 2013", Información y Estadísticas Sanitarias 2014, Madrid, 2014, p. 5. Disponible en versión digital: http://www.msssi.gob.es/estadEstudios/estadisticas/docs/Sist.Salud.UE 2013.pdf

${ }^{29}$ Cfr., http://www.europarl.europa.eu/charter/pdf/text es.pdf (Web del Diario Oficial de la Unión Europea, 18-12-2000, consultada en junio de 2016) 
FERNANDO H. LLANO ALONSO: IMPACTO DE LA MEDICINA GENÉTICA EN LOS DERECHOS DE LAS PERSONAS CON RIESGO DE TRANSMITIR ENFERMEDADES INCURABLES: UNA REVISIÓN CONCEPTUAL Y NORMATIVA

FECHA DE ENVÍO DE ORIGINAL: 23.11.2016 FECHA DE ACEPTACIÓN: 25.11.2016

DOI: http://dx.doi.org/10.12795/IETSCIENTIA.2016.i02.07

Como hemos comprobado al estudiar la regulación del DGP y la medicina procreativa en varios países europeos, unos Estados (España, Francia, Reino Unido y Portugal) permiten las pruebas con embriones con fines terapéuticos, aunque ello suponga la destrucción de los pre-embriones descartados para el implante en el útero materno, y otros en cambio (Alemania, Austria y Suiza) lo prohíben.

A propósito de estas discrepancias hermenéuticas surgidas entre los legisladores nacionales de los países miembros de la UE, en relación con la interpretación del alcance de la inviolabilidad de la dignidad humana y la indisponibilidad de la persona, nos encontramos ante la siguiente disyuntiva: el derecho a la salud y el bienestar del individuo a través de técnicas de ingeniería genética frente a un derecho de tercera generación tan complejo como el derecho al desarrollo de la personalidad de todo ser humano. En este sentido, como advierte Martha C. Nussbaum, conviene tener presente que los derechos humanos y las libertades de los individuos no son más que un desideratum hasta que los Estados de Derecho los convierten en una realidad tangible en forma de derechos fundamentales ${ }^{30}$.

Sin embargo, aunque son los gobiernos de los Estados de Derecho los que asumen la responsabilidad de garantizar el disfrute de los derechos y las libertades, e incluso éstos pueden delegar una parte de ese cometido en entidades privadas (ONG's, plataformas de voluntariado, etc.), hay ámbitos como el de la medicina genética- en los que la acción del Estado puede entrar en conficto con la libertad negativa de los individuos (por ejemplo, interfiriendo en la libertad reproductiva de los padres que desean realizar un DGP). Por otra parte, la inacción estatal en materia de medicina genética y derecho a la salud, puede causar situaciones de abuso o privación de derechos y libertades fundamentales (desde el derecho a la información hasta el consentimiento y la libre decisión del paciente). Según Nussbaum, en casos como estos, en los que se pone de manifiesto el profundo abismo que separa la teoría de la realidad de los derechos fundamentales es donde se hace necesario recurrir al "enfoque de las capacidades". El enfoque de las capacidades se centra en la protección de espacios de libertad tan cruciales que, de no existir, la vida no podría considerarse humanamente digna (de acuerdo con el principio kantiano que afirma que cada persona es un fin en sí misma) ${ }^{31}$.

De acuerdo con esta profesora de la Universidad de Chicago, el enfoque de las capacidades permitiría al Estado actuar en aras de la salvaguarda de los derechos y libertades de los ciudadanos (especialmente por motivos de salud pública) cuando se atente contra la dignidad de las personas (pacientes). En casos como este, en los que está en juego la inviolabilidad de la persona, el Estado estaría legitimado para invadir excepcionalmente los límites de privacidad de quien se ampara en en el ejercicio de la libertad negativa (esfera

\footnotetext{
${ }^{30}$ M. C. NUSSBAUM, Crear capacidades. Propuesta para el desarrollo humano, trad. esp: A. Santos Mosquera, Paidós, Barcelona, 2012, p. 87.

${ }^{31}$ lbid., pp. $52-55$ y 101.
} 
FERNANDO H. LLANO ALONSO: IMPACTO DE LA MEDICINA GENÉTICA EN LOS DERECHOS DE LAS PERSONAS CON RIESGO DE TRANSMITIR ENFERMEDADES INCURABLES: UNA REVISIÓN CONCEPTUAL Y NORMATIVA

FECHA DE ENVÍO DE ORIGINAL: 23.11.2016 FECHA DE ACEPTACIÓN: 25.11.2016.

DOI: http://dx.doi.org/10.12795/IETSCIENTIA.2016.i02.07

de privacidad del individuo que le protege de la intromisión de otros individuos o del Estado) y evitar que se vulnere la inviolabilidad moral de la persona y su indisponibilidad física ${ }^{32}$.

\section{Conclusiones}

Al término de este trabajo, pueden extraerse tres conclusiones generales:

En primer lugar, como se ha podido apreciar en el epígrafe 2, la investigación genética supone una mejora de las perspectivas de vida sana de una persona en cuyo ADN hay un componente genético que les hace susceptibles de desarrollar enfermedades incurables y transmitírselo a sus descendientes. A este respecto el ensayo clínico realizado sobre embriones humanos con fines terapéuticos plantea un evidente dilema ético en el que, de un lado, se trata de proteger la integridad del futuro feto, la vida del embrión incipiente y todo lo que en términos morales conlleva el concepto de dignidad humana, y de otro lado, se intenta garantizar el derecho de los padres a seleccionar genéticamente la composición genética de sus hijos para hacerlos más sanos. Adoptar uno u otro paradigma ético dependerá del concepto que tengamos de la inviolabilidad de la vida humana y de la indisponibilidad orgánica de la persona. No existe en la sociedad contemporánea un ethos que permita una interpretación unánime, y esto deja abierto el debate ético condicionado a la decisión que tomen con posterioridad los juristas y los políticos atendiendo al grado de consenso público y las necesidades que plantee la sociedad en un determinado momento. En todo caso, relativizar el sentido finalista de la dignidad humana como valor supremo puede abrir peligrosamente puertas a nuevas dimensiones éticas (que hoy serían consideradas anti-éticas) en las que se subvierta e instrumentalice el carácter sagrado de la persona como ser moral. Como advirtiera John Rawls, la base de la autoestima y la dignidad, en una sociedad justa, no puede ser la parte de los beneficios que correspondan a cada individuo, sino la distribución igualitaria y públicamente confirmada de los derechos y libertades de sus ciudadanos ${ }^{33}$.

En segundo lugar, desde un punto de vista jurídico, se abre también un debate entre los defensores del derecho a la vida del nasciturus e incluso del derecho al desarrollo de las futuras generaciones de personas anónimas aún no concebidas y los partidarios de garantizar el derecho a la salud y mejorar las perspectivas de vida sana a través de la medicina genética. En este sentido, como afirma Ronald Dworkin respecto a los conceptos jurídicos que -como los derechos humanos- están saturados de moral, tienen una extensión tan excesiva que resultan contraintuitivos, con lo que no solamente pierden su agudeza distintiva, sino su potencial crítico. Por eso, al entrar en colisión los derechos humanos, habría que establecer una clara diferencia entre los

\footnotetext{
${ }^{32}$ F. H. LLANO ALONSO, "El derecho al desarrollo en el sistema de derechos humanos: entre los derechos de la personalidad y la actividad del Estado", El derecho humano al desarrollo, ed: Mํㅣ. Garrido Gómez, Tecnos, Madrid, 2013, p. 66.

${ }^{33}$ J. RAWLS, A Theory of Justice, The Belknap Press of Harvard University Press, Cambridge (Massachusetts)-Londres, 1971, p. 544.
} 
derechos más firmemente arraigados en la sociedad y una ponderación de bienes de rango preferente o secundario ${ }^{34}$.

Finalmente, desde una perspectiva socio-política, se estima necesaria la presencia moderadora de la Administración Sanitaria como garante de los derechos fundamentales de los pacientes, llegando incluso a justificarse excepcionalmente la intervención del Estado en la esfera de libertad negativa de los individuos cuando lo que se halla en juego es son bienes jurídicos superiores como el derecho a la información del paciente, su libre consentimiento y su integridad física y moral. Se trata, en definitiva, de que el procedimiento de selección de bienes primariosde los ciudadanos -entre ellos la salud- no termine convirtiéndose en una operación tecnocrática de unos pocos (aquellos que por su posición aventajada dentro de la sociedad, están en disposición de desarrollar sus capacidades a costa de las de los demás) ${ }^{35}$.

\section{BIBLIOGRAFÍA CITADA}

ABELLÁN, F., "Aspectos bioéticos y legales del diagnóstico genético preimplantatorio (DGP)", Revista Iberoamericana de Fertilidad, 23/2006, pp. 99134.

ABELLÁN, F., Selección genética de embriones: entre la libertad reproductiva y la eugenesia, Comares, Granada, 2007.

ALKORTA IDIAKEZ, I., Regulación jurídica de la medicina reproductiva. Derecho español y comparado, Aranzadi, Cizur Menor (Navarra), 2003.

BLOCH, E., Derecho y dignidad humana, trad. esp: F. González Vicén, Aguilar, Madrid, 1980.

COLE-TURNER, R., "The Transhumanist Challenge", en Transhumanism and Trascendence: Christian Hope in an Age of Technological Enhancement (ed: R.

\footnotetext{
${ }^{34}$ R. DWORKIN, Los derechos en serio (1977), trad. esp: M. Guastavino, Ariel, Barcelona, 1984, pp. 77 y ss.

${ }^{35}$ A. SEN, Development as Freedom, Oxford University Press, Oxford, 1999, pp. 78-79 y 159.
} 
COLE-TURNER), Georgetown University Press, Washington D.C., 2011, pp. 118.

CROW, M. C., "Science, Technology and Democracy, en Beyond Humanism: Trans- and Post-humanism (ed: S. L. SORGNER). Vol III: Building Better Humans? Refocusing the Debate on Transhumanism (ed: H. TIROSHSAMUELSON and K. L. MOSSMAN), Peter Lang, Frankfurt am Main/Berlin/Bern/Bruxelles/New York/Oxford/Wien, pp. 13-30.

DIMOND, R., "Social and ethical issues in mitochondrial donation", British Medical Bulletin, 115 (1), 2015, pp. 173-182.

DWORKIN, R., Los derechos en serio (1977), trad. esp: M. Guastavino, Ariel, Barcelona, 1984.

HABERMAS, J., El futuro de la naturaleza humana ¿Hacia una eugenesia liberal?, trad. esp: C. S. Paidós, Barcelona-Buenos Aires-México, 2002.

HEIDEGGER, M., Tiempo y ser (1962), trad. esp: J. L. Molinuevo, Tecnos, Madrid, 1999.

LLANO ALONSO, F. H., "El derecho al desarrollo en el sistema de derechos humanos: entre los derechos de la personalidad y la actividad del Estado", El

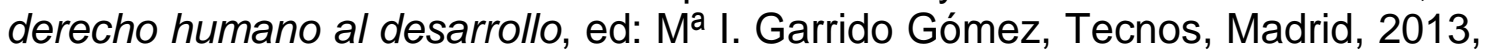
pp. 45-74.

LLANO ALONSO, F. H., "El hombre y la técnica en Ortega y Gasset", lus et Scientia, 1 (2015), pp. 1-24.

FRANKLIN, S. y ROBERTS, C., Born and Made. An Ethnography of Preimplantation Genetic Diagnosis, Princeton University Press, Princeton/Oxford, 2006.

KANT, I., Fundamentación de la metafísica de las costumbres (1785), trad. esp: M. García Morente, Espasa-Calpe, Madrid, 1921.

KANT, I., Crítica de la razón práctica (1788), trad. esp: E. Miñana y Villagrasa y M. García Morente, Sígueme, Salamanca, 1998.

NUSSBAUM, M. C., Crear capacidades. Propuesta para el desarrollo humano, trad. esp: A. Santos Mosquera, Paidós, Barcelona, 2012.

ORTEGA Y GASSET, J., "Meditación de la técnica" (1939), Obras completas (V), Fundación José Ortega y Gasset/Taurus, Madrid, 2004-2010, pp. 551-605.

PÉREZ LUÑO, A. E., "Kant y los derechos humanos", Historia de los derechos fundamentales. Tomo II: Siglo XVIII. Volumen II: La filosofía de los derechos humanos (Dir: G. PECES-BARBA MARTÍNEZ, E. FERNÁNDEZ GARCÍA y R. DE ASís ROIG), Dykinson-Instituto de Derechos Humanos "Bartolomé de Las Casas. Universidad Carlos III de Madrid, Madrid, 2001, pp. 435-495.

RAWLS, J., A Theory of Justice, The Belknap Press of Harvard University Press, Cambridge (Massachusetts)-Londres, 1971. 
IUS ET SCIENTIA (ISSN: 2444-8478) 2016, Vol. 2, nº 2, pp. 86-103

FERNANDO H. LLANO ALONSO: IMPACTO DE LA MEDICINA GENÉTICA EN LOS DERECHOS DE LAS

PERSONAS CON RIESGO DE TRANSMITIR ENFERMEDADES INCURABLES: UNA REVISIÓN CONCEPTUAL Y NORMATIVA

FECHA DE ENVÍO DE ORIGINAL: 23.11.2016 FECHA DE ACEPTACIÓN: 25.11.2016.

DOI: http://dx.doi.org/10.12795/IETSCIENTIA.2016.i02.07

SEN, A., Development as Freedom, Oxford University Press, Oxford, 1999.

SINGER, P., Una vida ética. Escritos, trad. esp: P. de Lora del Toro, Taurus, Madrid, 2002.

SPENGLER, O., El hombre y la técnica (1931), trad. esp: M. García Morente, Espasa-Calpe, Madrid, 1967.

WADDINGTON, C. H., "Epigenetics and evolution", en Symposia of the Society for Experimental Biology VII: Evolution, Cambridge University Press, Cambridge, 1953. 